\title{
APORÍAS, REALISMO Y OBJETIVIDAD: UNA NUEVA PERSPECTIVA DE FUNDAMENTACIÓN EPISTEMOLÓGICA EN TORNO A LA CIENCIA PRIMERA ARISTOTÉLICA
}

\author{
APORIAI, REALISM AND OBJECTIVITY: A NEW \\ EPISTEMOLOGICAL PERSPECTIVE OF FOUNDATION \\ AROUND THE ARISTOTELIAN FIRST SCIENCE
}

\author{
Julio Söchting Herrera ${ }^{1}$ \\ Universidad del Norte Santo Tomás de Aquino, Tucumán, Argentina
}

\begin{abstract}
Resumen: Desde la década de los 60's hasta la actualidad, las aporías aristotélicas han ocupado un lugar preponderante en la cuestión de la naturaleza epistemológica de la ciencia primera. Los autores varían desde una interpretación puramente dialéctica de las aporías a una interpretación adquisitiva de conocimiento. El presente artículo intenta mostrar la existencia de aporías objetivas, es decir, adquisitivas de conocimiento. Estas aporías están basadas en una teoría de la objetividad aristotélica que, superando la teoría de la contrariedad platónica, se expresa en su teoría de la contradicción, que funda la relación entre realidad y conocimiento en los distintos niveles de la estructura metafísica y lógica de la realidad: semántica, proposicional e inferencial. De este modo, las aporías objetivas son aquellas que expresan la determinación del objeto como sustancia y, desde ella, una posibilidad de conciliar el método diaporemático con el realismo epistemológico aristotélico.
\end{abstract}

Descriptores: Metafísica $\cdot$ Lógica $\cdot$ Semántica $\cdot$ Contrariedad $\cdot$ Contradicción

\begin{abstract}
From the 1960s to the present day, Aristotelian aporias have been at the forefront of the question of the epistemological nature of first science. Authors vary from a purely dialectical interpretation of the aporias to an acquisitive interpretation of knowledge. The present article attempts to show the existence of objective, i.e. acquisitive aporias of knowledge. These aporias are based on an Aristotelian theory of objectivity which, overcoming the Platonic theory of contrariety, is expressed in his theory of contradiction, which bases the relation between reality and knowledge on the different levels of the metaphysical and logical structure of reality: semantic, propositional and inferential. Thus, objective aporias are those that express the determination of the object as substance and, from it, a possibility of reconciling the diaporematic method with Aristotelian epistemological realism.
\end{abstract}

Keywords: Metaphysics $\cdot$ Logic $\cdot$ Semantics $\cdot$ Contrariety $\cdot$ Contradiction

Enviado: 19/04/2021. Aceptado: 03/06/2021.

\footnotetext{
${ }^{1}$ E-mail: juliosoch@gmail.com
} 


\section{INTRODUCCIÓN}

Desde la década de los 60's hasta entrado el siglo XXI, las aporías presentes en la Metafísica de Aristóteles, y en todo el Corpus, han suscitado un vivo interés en la interpretación de su filosofía en general y de la ciencia primera en particular. ${ }^{2}$ Este interés se debe a muchos factores, entre ellos: el temprano descubrimiento del carácter pedagógico del uso de los silogismos, es decir, no inventivo, sino expositivo (Scholz, 1975), ${ }^{3}$ la desconfianza heideggeriana en la metafísica escolar y deductiva (Aubenque, 2001), la valoración de la dialéctica como método teórico de adquisición de saber (Irwin, 1988, Cleary, 1995) y, sobre todo, la enorme cantidad de material diaporemático presente en el Corpus. Este interés, como es natural, se ha extendido a su fundamento histórico en Platón y Sócrates $^{4} \mathrm{y}$, también, aunque en menor grado, a su fundamento epistemológico, lo que nos lleva a los otros dos miembros del título de este breve artículo: el realismo y la objetividad.

En este artículo quisiera defender que en Aristóteles, particularmente, en su Metafísica, existen "aporías objetivas", que su estudio puede conducirnos a una noción aristotélica de "objetividad" y que esta noción no es una extrapolación impertinente y extemporánea, sino que, por el contrario, constituye una base de exploración válida para nuevas perspectivas de fundamentación epistemológica en Aristóteles basadas en sus avances en la teoría de la determinación y la contradicción.

\section{Aporía Y OBJETO}

En primer lugar, es necesario considerar que, en general, una aporía (desde su sentido etimológico primitivo hasta el sentido "técnico" aristotélico) puede ser definida, en general, como una oposición de premisas contrarias cuyo contenido proviene de la experiencia o del parecer de la comunidad científica reputada como tal. La aporía manifiesta una dificultad que, en el caso de las ciencias teóricas, consiste en la aparente imposibilidad de seguir adelante en la investigación o, al menos, con la carencia de "recursos" materiales o formales para llevarla a cabo. Las aporías objetivas pertenecen al ámbito del discurso mental (aporía tês dianoias) y manifiestan problemas "en las cosas mismas" (aporíai toû pràgmatos).$^{5}$

La semejanza entre las aporías subjetivas y objetivas es, claramente, el estado sicológico de escasez de conocimiento: la experiencia de estar "atados" o

${ }^{2}$ Para los textos de Met. hemos consultado y utilizado principalmente las ediciones de W. D. Ross, 1958 y de T. Calvo, 1998. Para Met. he seguido en todo a Calvo (1998), para el resto del Corpus a J. Barnes, 1991. Los textos griegos han sido obtenidos del útil instrumento digital de G. Reale, 2005.

${ }^{3}$ Doctrina que sigue, entre otros, Düring, 1990. Más amplio de criterio Ross, 1957.

${ }^{4}$ Cf. Motte y Rutten, 2001.

${ }^{5}$ Cf. Met. 3, 1. 
de no saber qué camino tomar en una encrucijada. La diferencia entre ambas radica en que el sabio y el científico "saben que no saben" respecto de algo y desean, en términos aristotélicos, saber. La investigación del campo semántico prâgma/ pragmateuomai en el Corpus me ha llevado a la convicción de que la categoría moderna "objetivo/objetividad" es pertinente para considerar esta diferencia. En efecto, prâgma se emplea, desde el punto de vista de la extensión del concepto, para denotar la sustancia concreta ("Sócrates"), el estado de cosas (como "Sócrates está sentado"), incluso la materia de un discurso, de una argumentación científica, jurídica, retórica y poética. En este sentido, "aporía objetiva” denota un problema en el cual la contrariedad de la denotación de las proposiciones en juego hace avanzar el intelecto hacia una solución (lusis).

El problema fundamental radica, entonces, en la comprensión de la teoría de la contradicción aristotélica, desarrollo al mismo tiempo que superación del núcleo de la dialéctica platónica: la oposición por contrariedad.

\section{ANTECEDENTES DEL PROBLEMA DE LA OPOSICIÓN}

El problema de la oposición de proposiciones se encuentra en el origen mismo del pensar filosófico y encuentra una de sus formulaciones más conocidas y, al mismo tiempo, más problemáticas en el Poema de Parménides. La afirmación del ser y la negación del no-ser son el criterio de demarcación del conocimiento de la ciencia que es la vía de la verdad en oposición a la vía de la opinión y del fenómeno que afirma ser lo que no es y no ser lo que es. ${ }^{6}$ La simple y elegante teoría eleática tenía, como evaluó Platón, una falla estructural mayor al momento de explicar el mundo fenoménico y, con él, el cambio. Existe un ámbito de realidad que es y no es al mismo tiempo: el reino de lo sensible opuesto al reino de lo inteligible. ${ }^{7}$

La teoría de las ideas, como es sabido, es una respuesta al desafío parmenídeo respecto del problema de la unidad y, por tanto, de la oposición. El mismo Platón, una vez estipulada su teoría estándar de las ideas, ${ }^{8}$ se vio en la necesidad de una reformulación crítica: por una parte, hubo de aceptar oposiciones ontológicamente originarias, como la Díada (con el complejo método para explicar desde ella la diversidad) y, al mismo tiempo indeterminar el no-ser como lo otro.

Esta estrategia fortaleció la oposición propia de los universales, dado que establecía mecanismos explicativos (la división dicotómica) y realizaba procesos

\footnotetext{
${ }^{6}$ Para una consideración panorámica e histórica respecto de la teoría de la oposición aristotélica, cf. Hamelin (1946), 163-85.

${ }^{7}$ Cf. Rep. 6, $508^{\mathrm{a}}-511^{\mathrm{d}}$.

${ }^{8}$ Esta teoría contiene dos elementos fundamentales: la ordenación jerárquica de lo real expresa en el símil de la línea dividida y la relación de esta jerarquía a través de las ideas de "participación" (méthexis) e "imitación" (mimêsis) que, por lo demás, Aristóteles considera ininteligibles.
} 
dialécticos de ascenso y de descenso desde los géneros mayores hasta las especies ínfimas (la definición de los individuos). Sin embargo, y al mismo tiempo, debilitaba radicalmente la oposición constitutiva de los individuos y su capacidad de ser considerados, por una parte, entes y por, otra, como objetos de conocimiento.

La crítica a la teoría de la oposición genérica de Platón constituye la expresión gnoseológica de la determinación ontológica aristotélica: la teoría de la oposición no genérica o contradicción. En lo que sigue, describiremos ambas teorías para mostrar, en su contraste, el valor del descubrimiento de la contradicción para la teoría de la objetividad aristotélica en general y para la tecnificación del método diaporemático en su aplicación a los problemas propios de la ciencia primera.

\section{LA TEORÍA DE LA CONTRARIEDAD PLATÓNICA}

La contrariedad platónica es la propiedad que poseen las especies que pertenecen a un mismo género. Son contrarios los extremos de una oposición genérica como el calor y el frío. Por esta razón, la contrariedad admite el más y el menos. Una sustancia, en cuanto perteneciente a un género, es sujeto de contrariedad y, por tanto, de variación: el agua puede recibir el calor o el frío, aunque no pueda poseer ambos al mismo tiempo.

Esta teoría es competente para la ciencia particular que evalúa la variación de los contrarios que son el límite de la definición y, por tanto, de la división del género en sus especies. Sin embargo, es incompetente para la determinación del individuo qua ente dado que, de aplicar el criterio de oposición, se obtiene la indeterminación de "lo otro" que es la conclusión del Sofista.

Para Aristóteles, aquí radica el problema de la ontología platónica crítica. ${ }^{9}$ La crítica de Platón a Parménides no ha podido ser completa dado que no ha tocado el principio fundamental que anima ambas ontologías: que el ser es un género y que, aunque universalísimo y trascendental, se comporta como tal.

Este principio genera una verdadera y propia indeterminación ontológica (tò héteron). Dada la intangibilidad epistemológica del individuo (sólo su definición última es posible por la división dicotómica) la experiencia de éste anula el conocimiento metafísico. Por el contrario, la afirmación metafísica hace imposible la conexión determinada del individuo con el ser que no sea por medio de la definición. Pero la definición ya excluye el carácter primero del ente. Nuevamente, y al igual que en el caso de Parménides, la objetividad de lo particular (o mejor, su no-objetividad) es yuxtapuesta a la objetividad de la dialéctica.

\footnotetext{
${ }^{9}$ El problema de la teoría platónica estándar era la suposición de formas separadas.
} 


\section{EL DESCUBRIMIENTO DE LA CONTRADICCIÓN}

La cuestión que ahora nos ocupa es central para la argumentación a favor de una teoría de la objetividad propiamente aristotélica y de la vinculación entre aporía y realismo, a saber, de la existencia de la propiedad por la cual las proposiciones determinadas significan verdad o falsedad.

Las proposiciones determinadas son afirmaciones y negaciones que poseen una misma referencia (prâgma). Inversamente, el prâgma tiene la propiedad de generar, a través de la determinación semántica, la composición y la división, proposiciones verdaderas y falsas.

Si el fundamento ontológico de la objetividad es la determinación, ¿cuál es su expresión gnoseológica e, incluso lógica? Aristóteles sostiene que se trata de una teoría de la oposición por contradicción perfectamente definida. ${ }^{10}$ Llamo perfectamente definida a aquella que se distingue claramente de la oposición por contrariedad.

El contexto de este descubrimiento es, como ya indicamos anteriormente, la polémica anti-sofística y la superación de la teoría de la contrariedad platónica. Prueba de lo primero es la referencia que hace Int. 7 a SE. 4, en la propia definición de contradicción. ${ }^{11}$ Prueba de lo segundo es el desarrollo, en contexto plenamente anti-platónico de la oposición de contrariedad y la oposición por contradicción en Met. 10, 4-7.

La teoría de la contradicción es formulada por Aristóteles como sigue: "es manifiesto que la negación de una sola afirmación es también una sola"12 o, más exactamente, "una sola afirmación se opone contradictoriamente a una sola negación". ${ }^{13}$

\section{EXPRESIÓN SEMÁNTICA DE LA CONTRADICCIÓN ARISTOTÉLICA}

La definición de contradicción y su distinción de la contrariedad ocurren en el amplio marco de la teoría de la proposición categórica descrita en Int. 1-6. En ese lugar, Aristóteles realiza una exposición constructiva de los elementos de la proposición a partir de las definiciones de 'nombre', 'verbo', 'frase' o 'aserción',

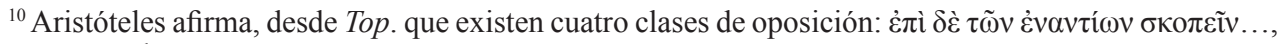

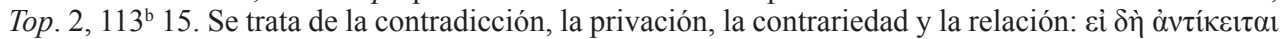

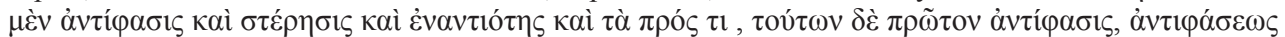

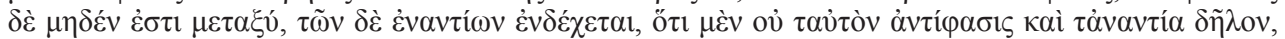
cf. Met. $10,4,1055^{\mathrm{a}}$.

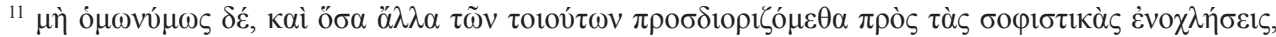

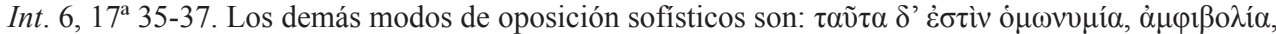

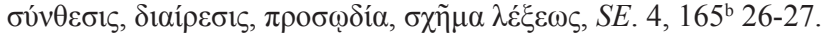

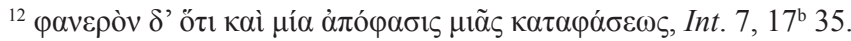

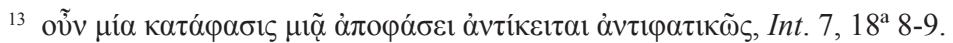


‘afirmación’ y ‘negación’. Con la teoría semántica descrita en estos términos, formula las definiciones de 'contrariedad' y 'contradicción' que expresan la objetividad de la teoría aristotélica de la proposición.

Luego de poner las bases ontológicas de la relación entre onoma y prâgma, ${ }^{14}$ Aristóteles comienza definiendo el 'nombre' (ónoma) como un sonido significativo (fônê sêmantikê) por convención que no significa tiempo y 'verbo' (rêma) como el nombre que "co-significa" (prossêmaînon) tiempo. ${ }^{15}$ Un dato importante para la teoría de la oposición que se está gestando es que Aristóteles considera que 'nohombre' y 'no está sano' no son, respectivamente, ni nombre ni verbo, dado que no significan nada determinado, por ello los denomina nombre indefinido (onoma aóriston) y verbo indefinido (aóriston rêma), respectivamente. ${ }^{16}$

La primacía ontológica del prâgma como ousía se manifiesta en la afirmación de que el verbo 'ser' no es un predicado real, es decir, no implica, por sí mismo, la realidad que co-significa: “en efecto, ni siquiera 'ser' o 'no-ser' es signo de la cosa real (tô̂ prágmatos), por más que se diga 'lo que es' a secas (psilon). En sí mismo, en efecto, no es nada, sino que co-significa una cierta composición, que no es posible concebir sin los componentes (tôn sugkeiménôn)" ${ }^{17}$

El enunciado asertivo o aserción es un sonido significativo alguna de cuyas partes es significativa por separado, pero no como afirmación. ${ }^{18}$ Existen aserciones simples y compuestas. ${ }^{19}$ La afirmación y la negación son aserciones compuestas que significan unión o separación de algo respecto de algo. ${ }^{20}$

Una vez llegado a este punto, Aristóteles establece el fundamento ontológico de la expresión semántica de la contradicción: "ahora bien, como es posible tanto aseverar que no se da lo que se da, como aseverar que se da lo que no se da, y de igual modo, respecto de los tiempos distintos del presente, cabría negar todo lo que uno afirmara y afirmar todo lo que negara; así que es evidente que a toda afirmación se le opone una negación y, a toda negación, una afirmación". ${ }^{21}$

\footnotetext{
${ }^{14}$ Cf. Int. 1.

${ }^{15}$ Cf. Int. 2-3.

${ }^{16}$ Cf. Int. 2, $16^{\mathrm{a}} 29-32$ e Int. 3, 16 ${ }^{\mathrm{b}} 11-15$.

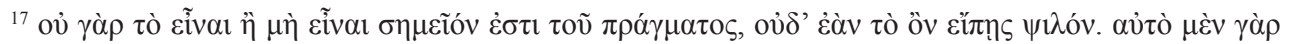

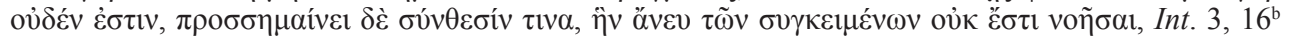
22-25.
}

${ }^{18}$ Cf. Int, $4,16^{\text {b } 26-28 . ~}$

${ }^{19}$ Cf. Int. 5.

${ }^{20} \mathrm{Cf}$. Int. 6.

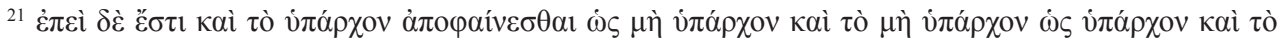

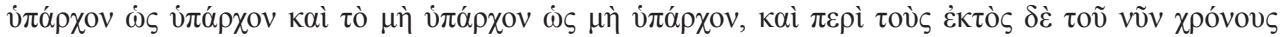

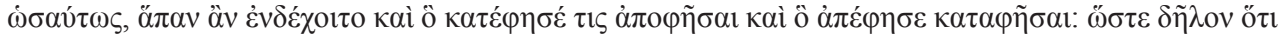

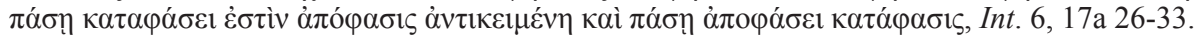


El "darse" (tò huparcheîn) denota la presencia del prâgma y, por tanto, del hupokéimenon necesario para fundar ontológicamente la oposición. Sin embargo, esta oposición, en tanto que indeterminada, puede afirmar o negar indistintamente, permaneciendo en el espacio de la dialéctica y abriendo flanco a la fundamentación semántica de la sofística. $^{22}$

La determinación de la oposición es la contradicción, por ello, inmediatamente después del pasaje antes citado, Aristóteles define: "y llamaremos contradicción a eso, a la afirmación y a la negación opuestas; digo que se oponen la [afirmación y la negación] de lo mismo acerca de los mismo, pero no de manera homónima, ni de ninguna de las otras maneras que distinguimos contra las distorsiones sofísticas". ${ }^{23}$

El género de la definición de "contradicción" (antífasis) es el "oponerse" (antikeimai). Se trata, ya desde aquí, de una imagen que podríamos denominar física y que representa el "estar uno frente a otro" de los que sostienen una discusión dialéctica. ${ }^{24}$ Sin embargo, esta imagen se precisa por la diferencia específica de la definición: la referencia a una y la misma cosa (tên toû autố katà tô̂ autoû), aludiendo, sin duda, al objeto (tô̂ prágmatos) de Int. 3, 16b 22-23 que, en ese contexto, fundamenta la referencia objetiva de la atribución categórica y, por tanto, su valor de verdad.

Esta determinación objetiva de la contradicción por el objeto es confirmada con la alusión a los modos sofísticos en los cuales es posible distorsionar la oposición uno de los cuales es mencionado: la homonimia. ${ }^{25}$

La contradicción es, pues, una propiedad de las proposiciones con referencia determinada, en el preciso sentido de objetiva. ${ }^{26}$

\footnotetext{
${ }^{22}$ Cf. $S E .3,165^{\mathrm{b}} 12-22$, donde Aristóteles enumera los cinco fines de la argumentación sofística: la refutación (elegchos), la falsedad (pseûdos), la paradoja (parádoxon), la incorrección (soloikismòs) y la locuacidad banal (tò poiêsai adoleschêsai tòn prosdialegómenon). Es posible que Aristóteles conceda a la sofística un cierto fundamento en el fenomenismo, como es posible ver en este pasaje, en $S E .1$ y en otros muchos de Met., sin embargo, su fundamento es semántico y radica en la oposición que se forma necesariamente entre la afirmación y la negación.

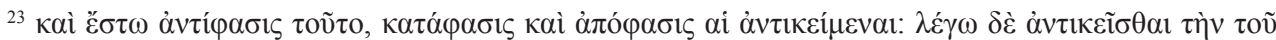

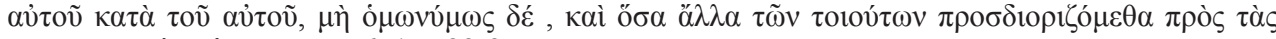

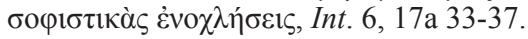

${ }^{24}$ Cf. Top. 1, 10, 104 $12-15$ donde se refiere la contradicción a la proposición dialéctica. En el mismo sentido, Top. 1, 15, $105^{\text {b }} 1-2$.

${ }^{25}$ Los demás modos son: la ambigüedad (amfibolía), la composición (súnthesis), la división (diaíresis), la acentuación (prôsôidia) y la forma de expresión (schêma léxeôs), cf. SE. 4, 165 23 -27. Respecto de

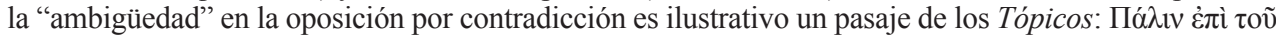

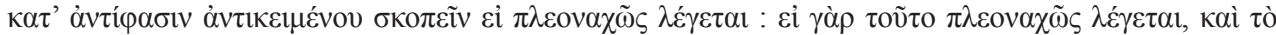

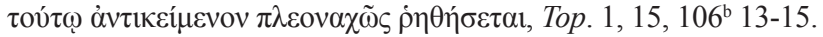

\footnotetext{
${ }^{26}$ Afirma Correia: “Aristóteles establece una teoría semántica de la contradicción. Define contradicción como un par de proposiciones que afirman y niegan lo mismo de la misma cosa. Normalmente, una contradicción se establece entre una afirmación y una negación que dicen lo mismo de la misma cosa. Con la expresión 'afirmar y negar lo mismo de la misma cosa' Aristóteles se refiere, según los comentarios antiguos de Int., a que no toda afirmación y negación harán una contradicción, sino sólo aquellas que afirman o niegan diciendo lo mismo de la misma cosa. Aquí, la expresión 'de la misma cosa' significa lo mismo que "con la misma referencia"' (Correia, 2003, 72). Una confirmación de esta doctrina en $S E$.

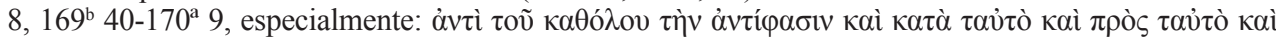
ஸ் $\alpha u ́ \tau \omega \varsigma, S E .8,170^{\mathrm{a}} 6-7$.
} 


\section{CONTRARIEDAD Y CONTRADICCIÓN}

Aristóteles establece una teoría definida de la contradicción porque está en condiciones de mostrar en qué se distingue la oposición de contrariedad de la oposición por contradicción antes descrita. Este paso es importante para la teoría de la objetividad ya que, según mi parecer, en Int. 7 Aristóteles emigra del frente puramente anti-sofístico ${ }^{27}$ y se traslada al mucho más fecundo frente anti-platónico.

Aparecen indicios de este paso en Top. 2, 8, donde Aristóteles discute los lugares comunes a partir de las formas de oposición, ${ }^{28}$ y en Int. 7 donde define la contrariedad y la contradicción, al mismo tiempo que establece el valor de verdad para cada una de las oposiciones.

Así, se da la "contrariedad": "si se asevera universalmente sobre lo universal que algo se da o no, habrá aserciones contrarias". ${ }^{29}$ La aserción contraria (enantía apofásei) se da en el contexto de "lo universal" (tò katholoù). El ejemplo es clásico: "todo hombre es blanco" se opone contrariamente a "ningún hombre es blanco". Lo universal es el contexto completo y definido de la objetividad platónica.

Junto a la "contrariedad" existe también la "contradicción": "se opone contradictoriamente (antikeîsthai) a la negación la afirmación que significa lo universal respecto a lo mismo que [la negación significa] de manera no universal (ou kathóulu)". ${ }^{30}$ El ejemplo es 'todo hombre es blanco" se opone a "no todo hombre es blanco'.

Esta distinción tiene un alto valor lógico y ontológico. Ambos inciden en la teoría de la objetividad que sostenemos. El valor lógico consiste en los valores de verdad que provienen de las inferencias inmediatas: la contradicción implica absoluta determinación de la verdad y de la falsedad de las oposiciones al mismo tiempo; la contrariedad implica la determinación de la verdad, pero no de la falsedad de las opuestas al mismo tiempo. ${ }^{31}$

El valor ontológico es que la determinación lógica de la contradicción y, en general, todas las inferencias del "cuadrado de las oposiciones" implican la

\footnotetext{
${ }^{27}$ Respecto de la descripción de este "frente" sofístico como personificado, cf. Dancy (1975), 59-73.

${ }^{28}$ Específicamente en Top. 2, 8, 113b 15-32, donde señala que las contradicciones deben ser examinadas de modo inverso a las implicaciones de modo que 'si el hombre es animal, entonces, el no-animal será no-hombre". Respecto de los contrarios, se debe mirar si el contrario "sigue al contrario", siguiendo las reglas de la implicación. Aristóteles no menciona ejemplo, pero es posible deducirlo del ejemplo dado a partir de la contradicción: 'si todo hombre es animal, entonces, algún hombre es animal", al mismo tiempo, es falso que 'todo hombre es no-animal'.

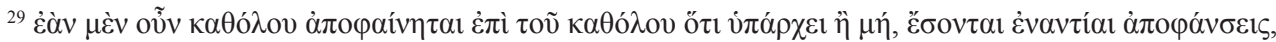
Int. $7,17^{\mathrm{b}} 3-5$.

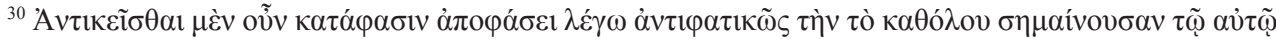

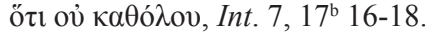

${ }^{31}$ Es lo que se denomina el "cuadrado de las oposiciones". Sobre estos valores de verdad afirma Correia: "[las contradictorias] no pueden ser verdaderas al mismo tiempo ni falsas al mismo tiempo. [Las contrarias] no pueden ser verdaderas al mismo tiempo, pero pueden ser falsas al mismo tiempo" (Correia, 2003, 75).
} 
posibilidad de, al mismo tiempo, determinar inequívocamente la proposición que denota el particular y a fortiori, el singular concreto (prâgma) respecto de su única proposición contradictoria que es siempre universal y; por otra, de definir, es decir, incluir genéricamente, éste mismo particular en una proposición universal.

El descubrimiento lógico de la contradicción, en síntesis, resuelve el problema metafísico del objeto dado que, al mismo tiempo, permite considerar el prâgma como hékaston y como ousía, en el sentido antes mencionado de hupokéimenon de atributos pertenecientes a un género y también, determinarlo como un tóde ti.

Este valor ontológico aparece claramente en Met. 4, donde Aristóteles formula el principio de no-contradicción tanto en términos de sujeto ${ }^{32}$ como en términos de prâgma: "y no será posible que una misma cosa (tò autò) sea y no sea sino por homonimia, como si al que nosotros llamamos 'hombre' otros lo llamaran 'no-hombre'. Pero la dificultad (tò aporoúmenon) no está en saber si es posible que una misma cosa sea (tò autò) y no sea simultáneamente en cuanto al nombre (tò ónoma), sino en realidad (tò prâgma)". ${ }^{33}$

\section{CONTRADICCIÓN Y OBJETIVIDAD ARISTOTÉLICA}

La clave de la comprensión de esta formulación del principio de No Contradicción (en adelante, PNC) y su vinculación con la teoría de la objetividad es clara en Met. 4, 4:

“es evidente (dêlon) que al menos esto es verdadero (alêthés): que las palabras 'ser' y 'no ser' significan algo determinado (todi) y, por tanto, no todo sería de este modo y no de este modo". 34

La objetividad radica en la determinación del prâgma. Esta determinación permite la definición como inclusión genérica. De este modo, es posible la ciencia particular desde la ciencia primera precisamente porque la objetividad particular (basada en la contrariedad) se fundamenta en la objetividad primera (basada en la contradicción).

Podemos observar el rendimiento de esta teoría en Met. 10, 2 donde Aristóteles enfrenta la aporía de la unidad ${ }^{35}$ y la resuelve desde la distinción

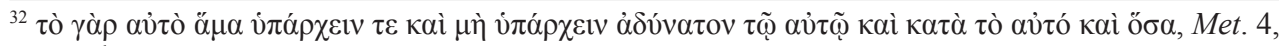
$3,1005^{\text {b }} 19-20$.

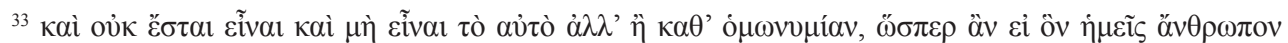

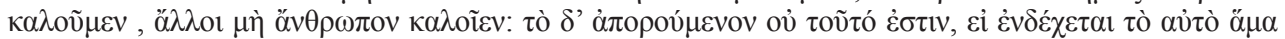

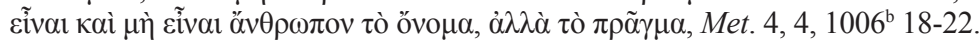

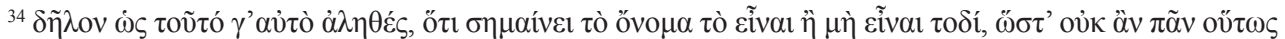

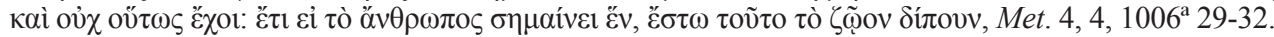

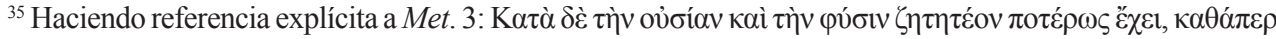

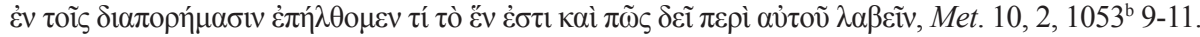


entre la oposición de contrariedad y la oposición por contradicción. En efecto, el problema de qué clase de unidad le conviene a la ousía, proviene del cruce de los dos tipos de objetividad antes mencionados. Por una parte, a la ousía le corresponde unidad genérica dado que, por su forma, pertenece a un género y es susceptible de contrariedad considerada en el universal. ${ }^{36}$ Por otra, la corresponde unidad numérica dado que se determina en la oposición entre uno y muchos. ${ }^{37}$

La contrariedad es definida aquí como "la diferencia máxima" (megistê diáfora) entre las cosas que pertenecen a un mismo género. ${ }^{38} \mathrm{~A}$ ella, por tanto, le pertenece el "término medio": un objeto puede ser caliente, tibio y frío.

La contradicción es definida como la oposición en la que no hay "término medio" (metaxú) $)^{39}$ lo que es claro desde la teoría de la contradicción expuesta en Int. 7. De este modo, la sustancia, considerada como un prâgma en su sentido primero es determinada por contradicción: entre su individualidad concreta (todi) y la proposición universal contradictoria no existe término medio y, por tanto, el fenomenismo físico y sofístico son insostenibles.

Sin embargo, la misma ousía es prâgma en el sentido de objeto de una proposición categórica universal porque es, a su vez, su esencia (tò ti ên ê̂nai). ${ }^{40}$ De este modo, Aristóteles supera la indeterminación del ser platónica en el Sofista porque puede ofrecer una teoría que explica, al mismo tiempo la definición y la determinación. ${ }^{41}$ La definición permite el conocimiento de la ciencia particular y su exposición en la demostración; la determinación permite el conocimiento de la ciencia primera y su expresión en el diaporêma, dado que su objeto no es demostrable $\mathrm{y}$, sin embargo, máximamente cognoscible.

Este doble modo epistemológico de objetividad (objetividad segunda/ particular y primera/metafísica) nos conduce a un problema que, desde la teoría que expongo parece encontrar una nueva solución: el problema del acceso al objeto primero, es decir, a la ousía como focalización del ente qua ente.

Antes de dar paso al problema del acceso cabe considerar aquí la crítica de Lukasiewicz al valor lógico y ontológico de PNC y, al mismo tiempo, la relativización de su formulación sicológica. ${ }^{42}$

\footnotetext{
${ }^{36}$ Cf. Met. $10,4$.

${ }^{37}$ Cf. Met. 10, 3, especialmente en la discusión respecto de "lo mismo".

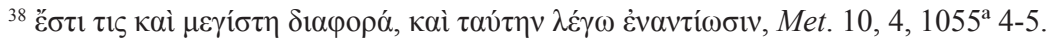

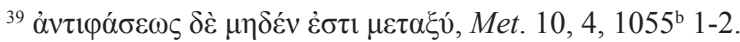

${ }^{40}$ Cf. Met. 7, 6, 1031 a 16-18.

${ }^{41}$ Esta explicación, como es natural no requiere la división dicotómica porque la determinación no proviene de los géneros mayores sino del individuo que siempre es accesible por experiencia (inducción) y nunca por pura deducción dialéctica, cf. An. Post. 2, 18 y An. 3, 7.

42 Para lo que sigue, cf. Lukasiewicz, 2000, 175-186. También Lukasiewicz, 1957 y 1979.
} 
Lukasiewicz considera que PNC aparece formulado en tres modos distintos: el modo ontológico, ${ }^{43}$ el modo lógico ${ }^{44}$ y el modo sicológico. ${ }^{45}$ El autor, para evitar ambigüedades, formula los distintos modos en términos "objetivos", definiendo 'objeto': “todo lo que es cualquier cosa que no es nada" y 'propiedad': "todo lo que se puede afirmar de un objeto". ${ }^{4}$

De este modo, define el modo ontológico como "algún objeto no puede a la vez poseer y no poseer una misma propiedad"; ${ }^{47}$ el modo lógico como "dos juicios, en los cuales uno le atribuye a un objeto justamente aquella propiedad que el otro le niega, no pueden ser verdaderos a la vez" ${ }^{48}$ y el modo sicológico como "dos convicciones, a las cuales le corresponden juicios contradictorios, no pueden existir a la vez en el mismo espíritu". ${ }^{49}$

Para Lukasiewicz, las dos primeras formulaciones son equivalentes y su pretensión de primeridad falsa, dado que existen otros principios lógicamente anteriores, como el principio de identidad y la definición de un juicio verdadero que, efectivamente, están a la base de la formulación de PNC que depende de la doble negación. ${ }^{50}$

Por su parte, la formulación sicológica es rechazada en su valor lógico porque todas las pruebas que Aristóteles utiliza (refutativas y apagógicas) incurren en las falacias de petición de principio y de ignoratio elenchii, es decir, están expuestas en el modo tollendo tollens que presupone PNC y porque todas las pruebas están dirigidas a recusar el juicio universal respecto de todos los objetos contradictorios y no cada uno de los particulares, a saber, que ciertos objetos son contradictorios. De este modo, PNC no tendría fundamento ontológico, carecería de valor lógico y expresaría un estado sicológico que tendría un valor práctico y ético porque constituiría un arma contra el error en el discurso. ${ }^{51}$

La argumentación de Lukasiewicz, que, en razón de brevedad, sólo hemos reseñado en este lugar, tiene una doble importancia. En primer lugar, establece la

${ }^{43}$ Cf. Met. 4, 3, 1005 $19-20$ y Met. 3, 2, $996^{\text {b }} 30$.

${ }^{44}$ Cf. Met. 4, 6, $1011^{\mathrm{b}} 13-15$ e Int. 6, $71^{\text {a }} 32-35$.

${ }^{45}$ Cf. Met. 4, 3, $1005^{\text {b } 23-26 . ~}$

46 “J'entends par object tout ce qui est quelque chose et qui n'est pas rien, et par propiété tout ce qu'on peut affirmer d'un object" (Lukasiewicz, 2000, 37).

47 “Aucun object ne peut à la fois posséder et ne pas posséder une même propiété" (Lukasiewicz, 2000, 48).

48 “Deux jugements, dont l'un attribue à l'object justement cette propiété que l'autre lui refuse, ne peuvent pas être vrais à la fois" (Lukasiewicz, 2000, 49).

49 "Deux convictions, auxquelles correspondent des jugements contradictoires, ne peuvent pas exister à la fois dans le même esprit" (Lukasiewicz, 2000, 50).

${ }^{50}$ Cf. Lukasiewicz, 2000, 176-7.

${ }^{51}$ Cf. Lukasiewicz, 2000, 181-4. 
noción de "objeto" como concepto primitivo para expresar todas las formulaciones de PNC en Aristóteles; la segunda, separa radicalmente, fruto del logicismo y el consecuente anti-psicologismo de su hora, el objeto ontológico y su expresión judicativa de la experiencia sicológica de la contradicción.

Respecto de lo primero, advertimos que el análisis de Lukasiewicz distingue de manera suficiente "objeto", "proposición" y "convicción" y en eso radica la fuerza de su argumentación: para él, los objetos lógicos son fregeanos: sustratos denotados por términos y funciones, que él denomina "propiedades". La convicción está absolutamente fuera de lugar de la argumentación lógica y, por ello, los argumentos por refutación no son, en absoluto, concluyentes.

Sin embargo, como hemos probado en el capítulo anterior, la noción de “objeto aristotélico" es compleja y contiene todos los niveles que Lukasiewicz separa: el objeto no es la cosa denotada simplemente, ni tampoco, la función de la cosa en el juicio. Se trata, por el contrario, del modo primero en el cual la realidad aparece como sujeto de propiedades y, por tanto, como capaz de permanecer en el alma formando una convicción ontológica.

La lógica y la ontología, como bien nota Lukasiewicz, no pueden separarse en Aristóteles. Pero esta no es razón para que PNC no tenga un valor ontológico fundamentativo. Por el contrario, si bien es cierto que PNC no es una ley lógica primitiva, no es menos cierto que su discusión y exposición está en el centro de lo que Aristóteles considera como las condiciones de posibilidad epistemológicas de la ciencia primera que no es lógica formal.

\section{APORÍA, OBJETIVIDAD Y REALISMO}

Desde el punto de vista objetivo, en el sentido que hemos dilucidado en esta investigación, el análisis lógico de las pruebas empleadas por Aristóteles no afecta la "objetividad" del principio y, por tanto, su valor ontológico. Es posible que Aristóteles no tuviera en mente la formulación de una teoría lógica formal perfectamente formada, como ha sido el intento de Whitehead y Russell desde el punto de vista lógico y de Meinong, desde el punto de vista de la "teoría del objeto". Lo que si es indiscutible es que la lógica de Aristóteles está comprometida con su ontología y que ese compromiso, específicamente, con la noción de sustancia ha permitido el desarrollo ulterior también de la lógica formalista. ${ }^{52}$

Este compromiso invariante es la determinación del objeto como sustancia $\mathrm{y}$, por tanto, con la posibilidad de establecer preguntas válidas respecto

\footnotetext{
${ }^{52}$ Se advierte la diferencia entre la noción misma de lógica que está en juego tanto para Lukasiewicz como para Aristóteles. Para el primero, la lógica fundamenta epistemológicamente la teoría de la ciencia; para el segundo, se trata de un instrumento para ella, sobre todo, en el orden expositivo.
} 
de los fundamentos del saber. El establecimiento de estas preguntas nos conduce, finalmente, al problema del acceso al objeto primero, es decir, lo que permanece como determinado tanto en la teoría de la ousía como en la teoría de los principios a partir de los cuales se realizan las demostraciones, entre los cuales se encuentra PNC, el "más firme de todos" no por su prioridad formalística lógica, sino por su prioridad ontológica que, teniendo un rendimiento sicológico y dialéctico (para la ética y la política), posee a su vez, valor lógico para una teoría de la deducción fundada en la sustancia.

En estas "aporías objetivas", referidas a objetos primeros indemostrables, parecen encontrarse nuevos caminos para comprender el realismo aristotélico no sólo como un "modo de pensar" el mundo sino como un "mundo" que se da al pensar en el permanente y adquisitivo juego que se suscita una y otra vez entre determinación y contradicción como fundamentos del saber.

\section{BIBLIOGRAFÍA}

Aubenque, P. 2001. "La función de la aporía en metafísica". (Dialéctica y Ontología). Seminarios de Filosofia, pp. 65-76 (Coloquio Internacional sobre Aristóteles. Santiago de Chile, Pontificia Universidad Católica de Chile).

Barnes, J., 1984. The Complete Works of Aristotle. The revised Oxford Translation. Edited by Jonathan Barnes, I-II, Bollingen Series LXXI-2: Princeton University Press.

Calvo, T. 1998. Aristóteles, Metafisica. Traducción, introducción y notas de Tomás Calvo Martinez. Madrid: Gredos

Cleary, J. 1994. "Phainomena in Aristotle's Methodology". International Journal of Philosophical Studies 2, pp. 61-97.

Correia, M. 2003. La lógica de Aristóteles. Lecciones sobre el origen del pensamiento lógico en la antigüedad. Santiago de Chile: Eds. Universidad Católica de Chile.

Dancy, R.M. 1975. Sense and Contradiction: A Study in Aristotle. Dordrecht-Holland/ Boston: Reidel.

Düring, I. 1956. Aristoteles. Darstellung und Interpretation seines Denkes. Heidelberg: Carl Winter. 
Hamelin, O. 1946. El sistema de Aristóteles, Buenos Aires: Estuario.

Irwin., T.H. 1988. Aristotle’s First Principles. Oxford: Clarendon Press.

Lukasiewicz, J. 1910. "Über den Satz des Widerspruchs bei Aristoteles", Bulletin International de l'Académie des Sciences de Cracovie, Classe d'historie et de philosohie, pp. 15-38 (reproducido en: Lukasiewicz, J. (1979), "Aristotle's on the Law of Contradiction", Barnes, J., Schofield, M., Sorabji, R. (eds.). Articles on Aristotle: 3. Metaphysics, St. Martin's Press, New York, pp. 50-62).

Lukasiewicz, J. 1957. Lukasiewicz, J., Aristotle's Syllogistic, Oxford: The Clarendon Press. (Versión en español: Lukasiewicz, J. 1975. La silogística de Aristóteles desde el punto de vista de la lógica formal moderna, Madrid: Tecnos).

Lukasiewicz, J. 2000. Du principe de contradiction chez Aristote, Paris: L'éclat.

Madigan, A. 1999. Aristotle Metaphysics, Books B and K 1-2, translated with a commentary by Arthur Madigan S.J. Clarendon Aristotle Series. Oxford: Clarendon Press.

Madigan, A. 2002. Aristotle on Aporia and the Possibility of Metaphysical Inquiry: Apuntes no publicados, facilitados generosamente por el profesor Madigan.

Motte, A. y Rutten, Chr. 2001. Aporia dans la philosophie grecque des origines à Aristote. Travaux du Centre d'Études Aristoteliciennes de l'Université de Liège édités par A. Motte et Chr. Rutten avec la collaboration de L. Bauloye et A. Lefka, Louvain-la-Neuve: Peeters.

Reale, G. 2005. Lexicon. Collana di lessici di filosofia antica. III. Aristóteles: Biblia.

Ross, W. D. 1958. Aristotle's Metaphysics, texto y comentario inglés. Oxford: Clarendon Press. 